\title{
THE RADIATION SOFT DYNAMIC RAM AS A PARTICLE DETECTOR
}

Patrick J. Winters

Naval Surface Weapons Center

White Oak, Silver Spring, Maryland 20910

\section{Abstract}

Radiation soft $64 \mathrm{~K}$ Dynamic Random Access Memory (DRAM) were obtained and tested for their response to known exposures of alpha-particles, and protons from the $n$-proton reaction in a hydrogenous foil. The memory was obtained from production lots not normally usable because of their soft error rate. The experimental system and sensitivities are described. The projected use of the device as a neutron detector by the Navy is examined.

\section{Introduction}

The fact that Dynamic Random Access Memory (DRAM) is sensitive to ionizing radiation is well known. ${ }^{1}$ Its use as a particle detector has been proposed, and several devices were studied or are under study. $2,3,4$ As dynamic memory densities have increased, the resulting sensitivity to radiation emitted from the packaging materials of the chip has been overcome only by careful circuit design, the use of purer packaging materials, and protective coatings to shield the surface of the circuit. This report concerns only the response of a commercially obtained circuit to alpha and proton radiation. ${ }^{3}$ Although the circuit was obtained commercially, it was manufactured in an early production run with no protective coating, and is not normally available. A task related to the stated one, but not covered here, concerns the conversion of neutrons to produce alpha or other charged particles.

A "soft" error occurs in dynamic memory when a stored data pattern has an error which can be corrected by rewriting the original pattern. The radiation sensitivity of dynamic memory should be proportional to the intensity of incident radiation as measured by the "soft" error rate. When a particular binary value is stored in dynamic memory, the memory cell written to will be placed into either a stable or metastable state. A cell in a metastable state will decay to the stable state in a minimum time of about 2 milliseconds. To prevent the loss of information each cell must be scanned and restored, if necessary. This process is called refreshing. Incident radiation which generates enough charge in or around a cell in the metastable state will cause the cell to flip to the opposite state. This causes an error in the stored data pattern that is "remembered." The rate at which these errors occur should be proportional to the intensity of particles striking the circuit.

This report consists of three parts: a discussion of some possible uses of the dynamic memory as a radiation detector, a description of the apparatus used to control and test the $64 \mathrm{~K}$ dynamic memory, and the results of two experiments which test the response of the $64 \mathrm{~K}$ dynamic memory to alpha-particles and protons.

\section{Possible Uses}

\section{Neutron Dosimetry}

The Navy uses tens of thousands of LiF thermoluminescent dosimeters (TLD). One of the reasons for selecting the LiF TLD for Neutron Dosimetry is the TLD's ability to detect dose equivalent exposures of a few mrem. Quality assurance requirements dictate that the TLD be transported to a central facility for reading. This process takes several weeks. The TLD will fade if it is exposed to elevated temperatures before it is read. Thus, uncertainties are introduced into the measured dose when a TLD is read due to unknown transit conditions.

A dynamic memory based dosimeter of sufficient sensitivity could have significant advantages over the present personnel dosimeter. It could be incorporated into a small package with radiation hard memory for local readout. With the dosages available immediately, investigation of unusual results could be resolved promptly, total doses automatically recorded and available for comparison with applicable standards.

\section{Electronic Systems Protection}

The Navy has requirements for the protection of critical electronic systems from failure due to a high flux of ionizing radiation. One way to reduce permanent failure is to remove power from the system while it is exposed. In order to accomplish this, a detector must be capable of very fast response times.

A radiation soft dynamic memory might yield meaningful counts in tens or hundreds of microseconds. The nearest competitors to such a device are radiation sensitive diodes. However, these diodes would not compete with the dynamic memory on a cost per sensitive area basis.

\section{The Experimental Apparatus}

\section{The 64K DRAM Controller}

Controlling the $64 \mathrm{~K}$ DRAM is not a simple task. Power, address, data, and control paths are provided on only 16 pins. Figure 1 shows the pin assignments of

\begin{tabular}{|c|c|c|c|}
\hline Nc 1 & \begin{tabular}{|l|l}
16 & vss \\
\end{tabular} & & ADDRESS INPUTS \\
\hline $\operatorname{DIN} 2$ & $15 \overline{C A S}$ & $A \phi-A 6$ & REFRESH INPUTS \\
\hline$\overline{\mathrm{WE}} 3$ & 14 DOUT & $\overline{\overline{W E}}$ & $\begin{array}{l}\text { READ/WRITE LEVEL } \\
\text { WN ADDRESS STROBE }\end{array}$ \\
\hline & 13 A6 & $\overline{\mathrm{RAS}}$ & ROW ADDRESS STROBE \\
\hline & $12 \mathrm{A3}$ & $\begin{array}{r}\text { DIN } \\
\text { DOUT }\end{array}$ & $\begin{array}{l}\text { DATA IN } \\
\text { DATA OUT }\end{array}$ \\
\hline$A z 6$ & 11 A4 & ucc & POWER (+5V) \\
\hline & 10 A5 & $\begin{array}{r}\text { uss } \\
\text { NC }\end{array}$ & $\begin{array}{l}\text { GROUND } \\
\text { NO CONNECTION }\end{array}$ \\
\hline $\operatorname{vcc} \sqrt{8}$ & 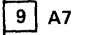 & & \\
\hline
\end{tabular}

FIGURE 1. THE PIN-OUT FOR THE 64K DRAM.

the circuit. The circuit consists of $2^{16}$ or 65,536 memory cell locations. Thus, a 16 bit address is needed to read or write to a particular cell. Addressing a location is accomplished by time multiplexing the address (AO-A7) in two 8-bit halves which are called the row address and column address. Two signals are used to latch each half of the address. They are the row address strobe (RAS) and the column address strobe (CAS). There is a path for data in (DI) and data out (DO) and a signal (WR) to determine which path is desired. For dynamic memory when binary information is written to a particular location, 
that memory cell will be placed into either a stable or a metastable state, depending on the circuit design. A cell in the metastable state will decay to the stable state in a minimum time of 2 milliseconds. To prevent the loss of information each cell must be refreshed. Refreshing is accomplished by using the RAS together with a refresh address (A0-A6) which is made to count modulo 128 for most designs. Thus, two rows of memory cells on each half of the circuit are refreshed simultaneously. A typical data sheet for this device has no fewer than 30 critical timing parameters. For this reason a $2-80$ based single board computer ${ }^{4}$ (SBC) which uses eight of the $64 \mathrm{~K}$ by 1 bit DRAM circuits as random access memory was chosen as a convenient controller in these initial tests.

The SBC also provides the hardware and software to communicate with the computer via a teletype and thus provides the means for executing simple commands such as filling memory with binary data and searching for binary data at particular memory locations. A drawback of this convenience is limited access to memory, as well as the lack of control over refresh rates and supply voltages. The $\mathrm{Z}-80$ has $64 \mathrm{~K}$ bytes in its memory space as shown in Figure 2. The resident

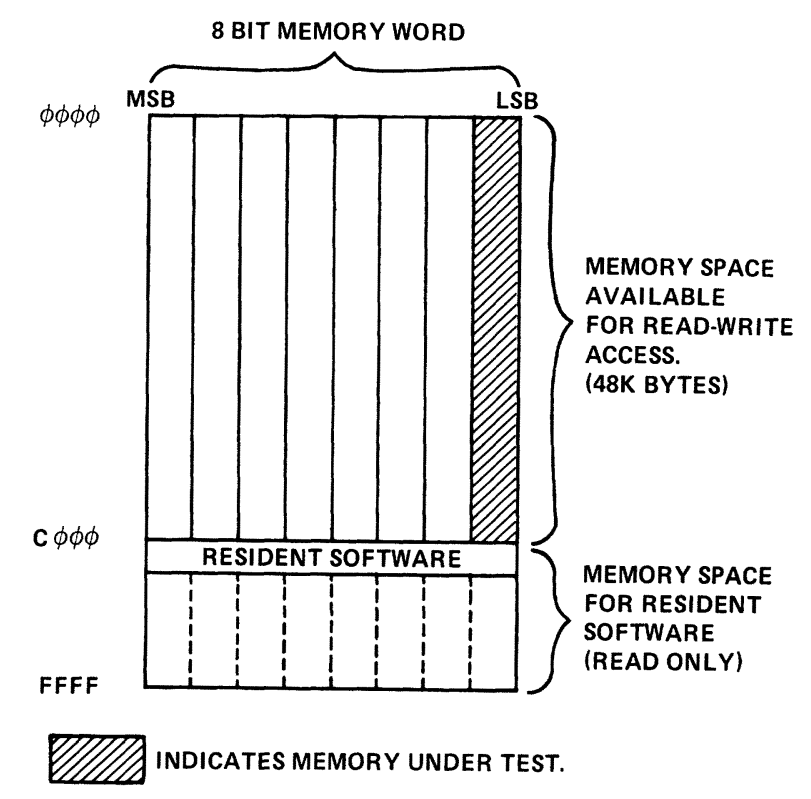

FIGURE 2. THE SINGLE BOARD COMPUTER MEMORY MAP.

software on the SBC occupies $\mathrm{ZK}$ bytes of the upper $16 \mathrm{~K}$ block of that space. Thus, only the first $48 \mathrm{~K}$ of memory is available for access by the resident software. The circuit under test was installed so as to occupy the least significant bit of the 8-bit data word.

\section{The Circuit Holder}

The memory circuit was attached to the SBC using a $50 \mathrm{~cm}$ twisted-pair cable with each pair consisting of a desired signal and ground. A small printed circuit card with a 16 pin socket was fashioned for holding the circuit. The cover plate for the portal of a vacuum chamber was modified to pass the 16 pins for accomodating vacuum conditions. Figure 3 shows the final configuration of the DRAM controller.

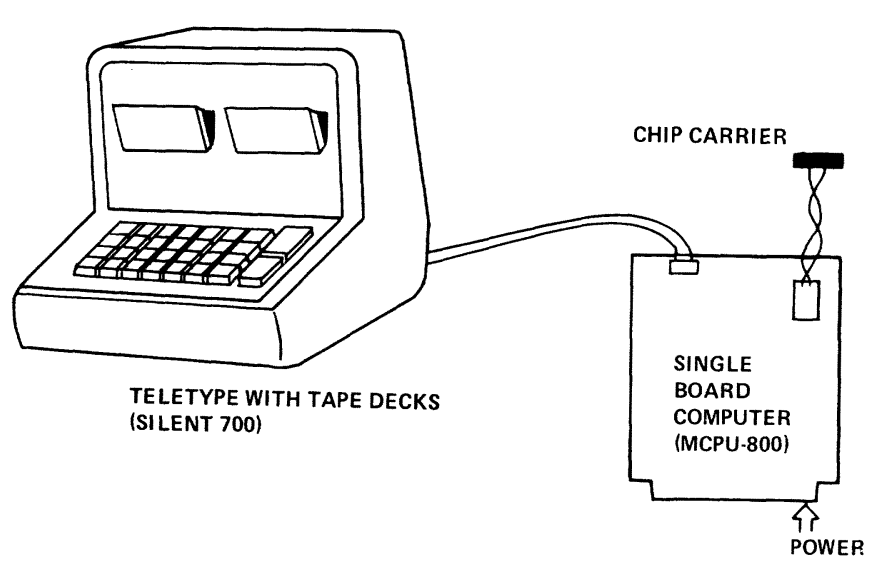

FIGURE 3. THE 64K DRAM CONTROLLER.

To determine if the circuit had a high natural soft error rate, the memory to be tested was installed, cleared (filled with binary zero) and run continuously (refresh only) for approximately 2 months. Two bit errors (binary ones) were recorded. Then the cover over the circuit was removed and replaced by clear adhesive tape. The exposed circuit appeared to be insensitive to normal room light and, in general, behaved in a much more rugged fashion than was anticipated.

\section{The Experiments}

\section{The Alpha-particle Experiment}

The $64 \mathrm{~K}$ DRAM was exposed to a $1 \mathrm{~mm}$ diameter collimated beam of $5.49 \mathrm{MeV}$ alpha-particles generated by $\mathrm{AM}^{241}$. The source was placed approximately $17 \mathrm{~mm}$ away from the surface of the circuit with the $1 \mathrm{~mm}$ collimating aperture one half the distance from source to target. The entire experiment was performed in a scatter chamber which served to provide a vacuum and an accurate means of aiming the beam. A surface barrier detector which is $100 \%$ efficient for the energetic alpha-particles was installed at an unused port of the scatter chamber and used to determine the strength of the incident beam. The beam was aimed so as to produce bit flips in the lower half of memory. The memory was filled with binary zeros and exposed during refresh only conditions. The bit errors (binary ones) and their locations were recorded on cassette tape. Then the memory was filled with binary ones and exposed again with refresh only conditions. The bit errors (binary zeros) and their locations were also recorded. The beam was then aimed at the surface barrier detector and the incident particle count rate was determined to provide the means for determining efficiency. Table 1 shows the results.

Table 1. Results of the Alpha-Particle Exposure

\begin{tabular}{lll}
$\begin{array}{l}\text { Bit Flip } \\
\text { Direction }\end{array}$ & $\begin{array}{l}\text { Rate } \\
(\mathrm{cpm})\end{array}$ & $\begin{array}{c}\text { Statistical } \\
\text { Error } \\
(\% \text { of Total) }\end{array}$ \\
\hline
\end{tabular}

$\begin{array}{lcc}0 \text { to } 1 & 1.98 & 2.3 \\ 1 \text { to } 0 & 1.33 & 2.3 \\ \begin{array}{l}\text { Surface } \\ \text { Barrier }\end{array} & 13.46 & 2.3 \\ \text { Detector } & & \end{array}$


Before this experiment was conducted, it was not anticipated that bit flips would occur in both directions. It was expected that either a stored zero or a stored one would place all the memory cells in an unstable or radiation sensitive state. When bit errors were encountered in both directions, the address locations of the bit flips were examined. It was found that in the first 256 locations of the memory space when a zero was stored initially, a random bit error was encountered in even locations. In the second 256 locations a random bit error occurred in odd locations. Conversely, when only ones were stored initially, random bit errors occurred at odd addresses of the first 256 locations and at even addresses for the second 256 locations. This pattern was then observed to repeat for each consecutive 512-bit block throughout the memory. However, exceptions in this pattern were observed. Approximately $3 \%$ of the random bit errors did not fit the pattern. All that was concluded from this experiment was that the circuit is $14.7 \%$ efficient for stored zeros and $9.9 \%$ efficient for stored ones with a probable combined efficiency for $5 \mathrm{MeV}$ alphaparticles of approximately $24 \%$. The pattern necessary to cause all the memory cells to be in a metastable or radiation sensitive state is complex. However, we must set the memory to this state to obtain maximum sensitivity.

\section{The Proton Response Experiment}

An experiment was conducted using a $\mathrm{Pu}-\mathrm{Be}$ calibration source and a $0.98 \mathrm{mg} / \mathrm{cm}^{2}$ polyethylene window to generate protons. The experiment was conducted on a calibration range used for exposing neutron survey instruments. Figure 4 shows the initial configuration.

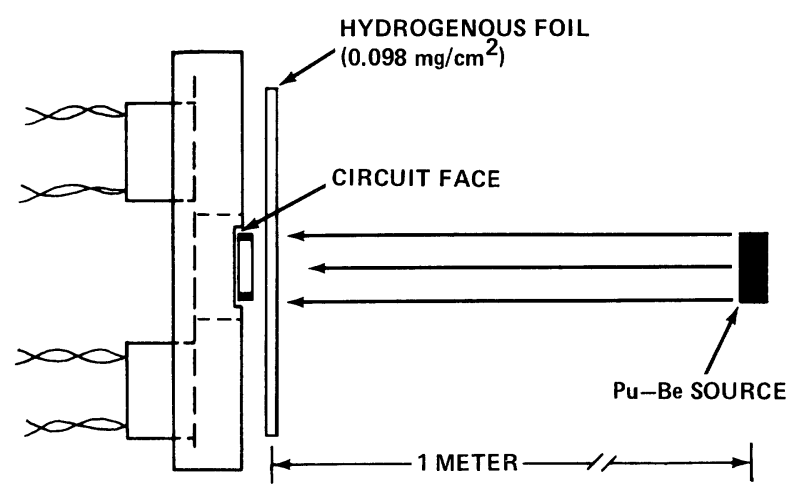

$\leftarrow$ TO CONTROLLER

FIGURE 4. ARRANGEMENT FOR THE PU-Be EXPOSURE

The Pu-Be calibration source produces a field of $16.1 \mathrm{mrem} /$ hour at a distance of 1 meter. The dynamic memory with the hydrogeneous foil overlay was first exposed for 120 minutes at the 1 meter distance with no errors in the memory recorded. The source was then placed at a distance of $20 \mathrm{~cm}$. The field strength was measured using a calibrated AN/PDR-70 neutron survey instrument. The measured field strength was 355 $\mathrm{mrem} /$ hour or $5.92 \mathrm{mrem} / \mathrm{minute}$. Four 2 hour exposures of the dynamic memory were conducted at this field strength with various combinations of overlays. The exposures and respective overlays consisted of: 1) the hydrogeneous foil alone, 2) the hydrogeneous foil, 3) the Ni foil alone, and 4) no foil. The Ni foil was used in exposure 2 to block protons emitted from the poly and thus determine if the recorded errors in exposure 1 were due to protons. Exposures 3 and 4 were made to determine background. Table 2 shows the results.

Table 2. Results of the 710 mrem Pu-Be Exposures with Various Overlays

$\begin{array}{clr}\text { Exposure } & \text { Overlay } & \text { Total } \\ 1 & \text { Poly } & 5 \\ 2 & \text { Poly, Ni } & 3 \\ 3 & \text { Ni } & 2 \\ 4 & \text { None } & 3\end{array}$

The calibration range was available for limited use and longer exposures were not possible. The results are inconclusive. It appears that all the total errors counted were probably due to direct neutron or gamma interactions with the dynamic memory. The refresh rate used in this experiment was 6 times faster than necessary. Slower refresh rates may improve proton sensitivity.

\section{Summary}

The mechanisms by which the $64 \mathrm{~K}$ dynamic memory responds to incident ionizing radiation is not well understood. However, the circuit will detect alphaparticles of sufficient energy (5.49 MeV). The circuit did not respond to protons emitted from a hydrogenous foil exposed to a $\mathrm{Pu}-\mathrm{Be}$ neutron source. It did show a small response which was attributed to direct neutron or gamma interaction. Future experiments using the dynamic memory at reduced refresh rates and voltages will be conducted to see if the response to protons can be improved. Still to be considered is the classical problem of relating the charged particle response of the possible new detector to the neutron dose equivalent. Methods for providing this relationship are being formulated. They include using the n-alpha reaction as an analog of the LiF TLD, and the N-proton reaction for detecting fast neutrons. When the response of the dynamic memory to these charged particles is well understood, experiments will be conducted to test these formulations. Acknowledgements

The author would like to thank Drs. G. Riel and D. Simons of the Naval Surface Weapons Center for their discussions and assistance in conducting these experiments. Appreciation is also given to $\mathrm{H}$. Cordova and P. Cady for their help in assembling the apparatus of the experiments and to J. Madello for preparation of the manuscript.

\section{References}

1. T.C. May, and M.H. Woods, "Alpha Particle Induced Soft Errors in Dynamic Memories," IEEE Trans. Elect. Dev. ED-26, 1979, pp. 2-16.

2. G. Cerofolini and G. Ferla, "A Proposal: Dynamic Rams as Particle Detectors," Nuclear Inst. and Meth. 169, 1980, pp. 125-128.

3. R.C. Wyatt, P.J. McNulty, and P. Toumbas, "Soft Errors Induced By Energetic Protons," IEEE Trans. Nuc1. Sci. NS-26, 6, 1979, pp. 4905-4917.

4. J. Davis, P. Winters, and B. Houston, "Progress on the Digital Dosimeter," NSWC TR-82-456, to be published.

5. MCM 6665; Manufacturer: Motorola.

6. MCPU-800; Manufacturer: Miller Technology, Los Gatos, CA 95030. 